\title{
A note on the robustness of PBIBD(2)s against breakdown in the event of observation loss
}

\author{
J. D. Godolphin ${ }^{1}$ \\ University of Surrey
}

\begin{abstract}
Summary
Robustness against design breakdown following observation loss is investigated for Partially Balanced Incomplete Block Designs with two associate classes (PBIBD(2)s). New results are obtained which add to the body of knowledge on PBIBD(2)s. In particular, using an approach based on the E-value of a design, all $\operatorname{PBIBD}(2)$ s with triangular and Latin square association schemes are established as having optimal block breakdown number. Furthermore, for group divisible designs not covered by existing results in the literature, a sufficient condition for optimal block breakdown number establishes that all members of some design sub-classes have this property.

Key words: concurrence; connectivity; design breakdown; E-value; information matrix
\end{abstract}

\section{Introduction}

Let $\mathcal{D}_{v, b, k}$ denote the class of binary equireplicate connected designs in $v$ treatments, arranged in $b$ blocks of size $k$. The common treatment replication is $r=b k / v$. When they exist, Balanced Incomplete Block Designs (BIBDs) have universal optimality in their respective $\mathcal{D}_{v, b, k}$. However, most $\mathcal{D}_{v, b, k}$ classes contain no BIBDs and, for these classes, $\operatorname{PBIBD}(2)$ s can provide useful designs. Two treatments in a $\operatorname{PBIBD}(2)$ are first associates, and occur together in $\lambda_{1}$ blocks, or are second associates and occur together in $\lambda_{2}$ blocks $\left(\lambda_{1} \neq \lambda_{2}\right)$. A PBIBD(2) is summarised by the parameter set $\left(v, b, r, k ;\left(\lambda_{1}, \lambda_{2}\right)\right)$. It is the flexibility afforded by the two concurrences, $\lambda_{1}$ and $\lambda_{2}$, that gives rise to the existence of PBIBD(2)s in many $\mathcal{D}_{v, b, k}$ classes which contain no BIBD. This makes the designs useful in a wide variety of experimental situations. For example see Bose et al. (2013), Kuhn and Kiefer (2013) and Best et al. (2011) for applications of PBIBD(2)s in key predistribution in sensor networks, and in trials involving educational assessment and sensory evaluation.

Clatworthy (1973) gave extensive tables of $\operatorname{PBIBD(2)s,~all~with~} k$ and $r$ in the range $[2,10]$. Since the publication of these tables, constructions for numerous additional

1 Department of Mathematics, University of Surrey, Guildford, Surrey. GU2 7XH, UK Email: j.godolphinesurrey.ac.uk 
PBIBD(2)s have appeared in the literature. See for example: Cheng, Constantine \& Hedayat (1984), Sinha (1987), Greig, Kreher \& Ling (2002), Henson, Sarvate \& Hurd (2007) and Hurd \& Sarvate (2008).

There can be considerable variation in $\operatorname{PBIBD}(2)$ properties even within a given $\mathcal{D}_{v, b, k}$. Designs in the $\operatorname{PBIBD}(2)$ class which are as close to BIBDs as possible, in that $\left|\lambda_{1}-\lambda_{2}\right|=1$, tend to have 'good' properties, with many being optimal in their $\mathcal{D}_{v, b, k}$, with respect to various criteria. See Conniffe \& Stone (1975), Cheng (1978), Cheng (1980), Constantine (1982) and Cheng \& Bailey (1991), among others. Less attention has been given to $\operatorname{PBIBD}(2)$ s with $\left|\lambda_{1}-\lambda_{2}\right|>1$ : some are known to have low efficiency.

It is common for some observations corresponding to a planned design to be missing. For example, crops can be spoilt through drought or flood; human error can lead to observation loss; funding cuts can cause experiments to be concluded prematurely. Depending on the circumstances, observation loss can involve loss of individual observations, or of entire blocks. The most extreme consequence occurs if all observations in one or more treatments are lost, or if the eventual design is disconnected. In either case, breakdown is said to have occurred (see Mahbub Latib, Bretz \& Brunner 2009) and some treatment contrasts will be inestimable. This is generally disastrous for the objectives of the experiment. Therefore, in experimental situations where observation loss can occur, consideration of robustness should be included in the design selection process.

A design in $\mathcal{D}_{v, b, k}$ is robust against the loss of $t$ blocks if all treatment contrasts are estimable from every eventual design resulting from loss of $t$ blocks. Robustness against the loss of individual observations is similarly defined. The block breakdown number, $t_{*}$, of a design is the number of blocks that need to be lost before the possibility of design breakdown arises. It follows that $t_{*} \leq r$. A design with $t_{*}=r$ is robust against the loss of any $r-1$ blocks and is said to have optimal block breakdown number.

A design which is robust against the loss of any $t$ blocks is also robust against the loss of any $t$ observations. However, the converse is not true. Consider the following design, $D \in \mathcal{D}_{10,10,4}$, which has $r=4$ and is robust against the loss of any three observations.

\begin{tabular}{cccccccccc}
\multicolumn{10}{c}{ Block } \\
1 & 2 & 3 & 4 & 5 & 6 & 7 & 8 & 9 & 10 \\
\hline 1 & 1 & 1 & 2 & 6 & 6 & 6 & 7 & 1 & 3 \\
2 & 2 & 3 & 3 & 7 & 7 & 8 & 8 & 2 & 4 \\
3 & 4 & 4 & 4 & 8 & 9 & 9 & 9 & 6 & 8 \\
5 & 5 & 5 & 5 & 10 & 10 & 10 & 10 & 7 & 9
\end{tabular}


Design $D$ does not have optimal block breakdown number. The design is robust against the loss of any one block, but the loss of blocks 9 and 10 results in design breakdown, since no comparisons can be made between a treatment in set $\{1,2,3,4,5\}$ and one in $\{6,7,8,9,10\}$ from the remaining eight blocks. Thus $D$ has $t *=2<r$. This demonstrates that having optimal block breakdown number is a stronger robustness property than the property of being robust against the loss of $r-1$ observations.

Ghosh (1982) established that all BIBDs have $t_{*}=r$. For binary incomplete block designs, not necessarily with equal treatment replication, robustness against the loss of whole blocks was investigated by Baksalary and Tabis (1987), Sathe and Satam (1992), Godolphin \& Warren (2011) and Godolphin (2016). Bailey, Schiffl \& Hilgers (2013) and Tsai \& Liao (2013) focussed attention on robustness of designs with blocks of size two. There is very little robustness work that is specific to PBIBD(2)s. Ghosh, Rao, \& Singhi (1983) proved that, with the exception of a sub-class of group divisible designs, all $\operatorname{PBIBD}(2)$ s are robust against the loss of $r-1$ observations, but these authors did not consider robustness against the loss of whole blocks. Godolphin \& Godolphin (2015) developed a lower bound for $t_{*}$ for a $\operatorname{PBIBD}(2)$. The bound is of limited use for general inference because it depends on the calculation of a value specific to each $\operatorname{PBIBD}(2)$.

This work makes a significant contribution to the knowledge on $\operatorname{PBIBD}(2)$ s by establishing that all designs in some categories of $\operatorname{PBIBD}(2)$ have optimal block breakdown number. Furthermore, for the only sub-class of the group divisible designs containing some members that do not have optimal block breakdown number, simple conditions for $t_{*}=r$ are developed.

The paper is organised as follows. Section 2 contains preliminary results. In Section 3, bounds based on the E-value are used to establish $t_{*}=r$ for all designs in two categories of $\operatorname{PBIBD}(2)$, and a condition is given for optimal breakdown number for regular group divisible designs with $\lambda_{1}>\lambda_{2}$. As a corollary, all regular designs with $\lambda_{1}-\lambda_{2}=1$ are shown to have $t_{*}=r$. Since this design sub-class contains designs known to be optimal with regards to various criteria and has concurrences differing only by one, it is an appealing source of designs if a BIBD is not available in a specific $\mathcal{D}_{v, b, k}$. The fact that all designs in the subclass have optimal block breakdown number and thus have robustness properties which match those of BIBDs was not previously known, and adds to their appeal as useful alternatives to BIBDs.

Every design in a $\mathcal{D}_{v, b, k}$ with $v<2 k$ has $t_{*}=r$. See Theorem 1 of Godolphin \& Godolphin (2015) for details. Therefore, it is assumed throughout that $v \geq 2 k$. 
Let $D_{0} \in \mathcal{D}_{v, b, k}$. Then $D_{0}$ has $v \times b$ incidence matrix $N$, with $(i, j)$ entry unity if treatment $i$ occurs in block $j$ and $(i, j)$ entry zero otherwise. The design $D_{0}$ has information matrix

$$
C=r I-\frac{1}{k} N N^{\top}
$$

where $I$ is the $v \times v$ identity matrix and $N^{\top}$ is the transpose of $N$. The E-value of $D_{0}$ is the smallest positive eigenvalue of $C$, denoted by $\mu$. A design in $\mathcal{D}_{v, b, k}$ with largest E-value is E-optimal in the class. Godolphin (2016) derived a lower bound for $t_{*}$ in terms of $\mu$ :

$$
\min \{\lceil\mathcal{T}\rceil, r\} \leq t_{*}
$$

where $\lceil x\rceil$ denotes the ceiling of $x$ and

$$
\mathcal{T}=\frac{8 \mu k^{2}(v-k)}{v\left(2 k^{2}-1+(-1)^{k}\right)} .
$$

In this work, $D$ is taken to be a $\operatorname{PBIBD}(2)$ in $\mathcal{D}_{v, b, k}$. Each treatment has $n_{1}$ first associates and $n_{2}$ second associates. Fundamental identities are:

$$
v r=b k, n_{1}+n_{2}=v-1
$$

$$
r(k-1)=n_{1} \lambda_{1}+n_{2} \lambda_{2} .
$$

Full specification of the association structure of $D$ requires further parameters: $p_{j k}^{i}$, for $i, j, k=1,2$, with $p_{j k}^{i}=p_{k j}^{i}$. For any two treatments which are first associates, there are $p_{j k}^{1}$ treatments which are $j$ th associates of the first treatment and $k$ th associates of the second treatment. Parameters $p_{j k}^{2}$ are similarly defined for pairs of treatments which are second associates. These additional parameters are typically displayed in matrix form:

$$
P_{1}=\left[\begin{array}{ll}
p_{11}^{1} & p_{12}^{1} \\
p_{21}^{1} & p_{22}^{1}
\end{array}\right], \quad P_{2}=\left[\begin{array}{ll}
p_{11}^{2} & p_{12}^{2} \\
p_{21}^{2} & p_{22}^{2}
\end{array}\right] \text {. }
$$

The non-zero eigenvalues of the information matrix of $D$ take exactly two values, $\mu_{1}$ and $\mu_{2}$. Connor \& Clatworthy (1954) obtained expressions for these in terms of design parameters:

$$
\mu_{1}=\frac{2 r(k-1)+\left(\lambda_{1}-\lambda_{2}\right)(-\gamma+\sqrt{\Delta})+\lambda_{1}+\lambda_{2}}{2 k}
$$

and

$$
\mu_{2}=\frac{2 r(k-1)+\left(\lambda_{1}-\lambda_{2}\right)(-\gamma-\sqrt{\Delta})+\lambda_{1}+\lambda_{2}}{2 k}
$$


where $\gamma=p_{12}^{2}-p_{12}^{1}$ and $\Delta=\gamma^{2}+2 p_{12}^{1}+2 p_{12}^{2}+1$. Thus $D$ has $\mu=\mu_{1}$ if $\lambda_{1}<\lambda_{2}$ and $\mu=\mu_{2}$ if $\lambda_{1}>\lambda_{2}$.

\section{Robustness properties of some categories and sub-classes of $\operatorname{PBIBD(2)}$}

All triangular designs and all designs with a Latin square association scheme will now be shown to have $t_{*}=r$. A sufficient condition is developed for a sub-class of the group divisible designs to have this property.

\subsection{Triangular association scheme}

Triangular designs form a $\operatorname{PBIBD}(2)$ category with $v=n(n-1) / 2$ treatments, for some integer $n \geq 5$. The treatments are arranged into $n$ sets of $n-1$ treatments such that each treatment occurs in two sets and each pair of sets has one common treatment. Any two treatments occur together in at most one set: treatments occurring together in a set are first associates, and treatments not occurring together in any set are second associates. Clatworthy (1973) gave 100 triangular designs with $r, k \leq 10$. Cheng, Constantine \& Hedayat (1984) and Greig, Kreher \& Ling (2002) used graphical constructions for several series of triangular designs.

From the association structure it follows that $n_{1}=2(n-2)$ and $n_{2}=(n-2)(n-$ $3) / 2$. To complete the association structure:

$$
P_{1}=\left[\begin{array}{cc}
n-2 & n-3 \\
n-3 & (n-3)(n-4) / 2
\end{array}\right], \quad P_{2}=\left[\begin{array}{cc}
4 & 2 n-8 \\
2 n-8 & (n-4)(n-5) / 2
\end{array}\right] .
$$

Use of (5), (6) and (7) gives, $\mu_{1}=\left\{r(k-1)+2 \lambda_{1}-\lambda_{2}\right\} / k$ and $\mu_{2}=\{r(k-1)-(n-$ 4) $\left.\lambda_{1}+(n-3) \lambda_{2}\right\} / k$.

Theorem 1. Every $\operatorname{PBIBD(2)}$ with triangular association scheme has optimal block breakdown number.

Proof: The approach that is applied in this proof focusses on a lower bound for $\mathcal{T}$. The cases $\lambda_{1}<\lambda_{2}$ and $\lambda_{1}>\lambda_{2}$ are considered separately.

For $\lambda_{1}<\lambda_{2}$, the E-value is $\mu=\mu_{1}=\left\{r(k-1)+2 \lambda_{1}-\lambda_{2}\right\} / k$. From (2),

$$
\begin{aligned}
\mathcal{T} & =\frac{8\left\{r(k-1)+2 \lambda_{1}-\lambda_{2}\right\} k\{n(n-1)-2 k\}}{n(n-1)\left(2 k^{2}-1+(-1)^{k}\right)} \\
& \geq \frac{4 r(k-1)\{(n-2)(n-3)-2\}\{n(n-1)-2 k\}}{k n(n-1)(n-2)(n-3)}, \\
& =\frac{4 r\{n(n-1)+2-2 k-n(n-1) / k\}(n-4)}{n(n-2)(n-3)},
\end{aligned}
$$


using $2 k^{2}-1+(-1)^{k} \leq 2 k^{2}, \lambda_{1} \geq 0$, and $\lambda_{2} \leq 2 r(k-1) /\{(n-2)(n-3)\}$ from (4). For values of the block size $k$ within the range $[2, v / 2=n(n-1) / 4]$, the maximum value of $2 k+n(n-1) / k$ is $4+n(n-1) / 2$, which is achieved for $k=2$ and for $k=n(n-1) / 4$, when the latter term is an integer. Thus,

$$
\mathcal{T} \geq \frac{2 r\left(n^{2}-n-4\right)(n-4)}{n(n-2)(n-3)}>\frac{2 r(n-4)}{n-3}>r .
$$

$$
\begin{aligned}
& \text { For } \lambda_{1}>\lambda_{2}, \mu=\mu_{2}=\left\{r(k-1)-(n-4) \lambda_{1}+(n-3) \lambda_{2}\right\} / k \text {. From (2), } \\
& \qquad \begin{aligned}
\mathcal{T} & =\frac{8\left\{r(k-1)-(n-4) \lambda_{1}+(n-3) \lambda_{2}\right\} k\{n(n-1)-2 k\}}{n(n-1)\left(2 k^{2}-1+(-1)^{k}\right)} \\
& \geq \frac{2 r(k-1)\{2(n-2)-(n-4)\}\{n(n-1)-2 k\}}{k n(n-1)(n-2)}, \\
& =\frac{2 r\{n(n-1)+2-2 k-n(n-1) / k\}}{(n-1)(n-2)},
\end{aligned}
\end{aligned}
$$

using $2 k^{2}-1+(-1)^{k} \leq 2 k^{2}, \lambda_{2} \geq 0$ and, from (4), $\lambda_{1} \leq \frac{r(k-1)}{2(n-2)}$. As above, the maximum value of $2 k+n(n-1) / k$ is $4+n(n-1) / 2$. Thus,

$$
\mathcal{T} \geq \frac{r\left(n^{2}-n-4\right)}{(n-1)(n-2)}>r
$$

Thus, for both $\lambda_{1}<\lambda_{2}$ and $\lambda_{1}>\lambda_{2}$, it has been shown that $\mathcal{T}>r$. Hence, $t_{*}=r$ from (1), establishing that all $\operatorname{PBIBD}(2)$ s with triangular association scheme have optimal block breakdown number.

\section{2. $L_{i}(n)$ Latin square association scheme}

For $v=n^{2}$ treatments, with $n \geq 3$, the existence of an $L_{i}(n)$ association scheme depends on there being $i-2$ mutually orthogonal $n \times n$ Latin squares. The parameter $i$ is also constrained by $2 \leq i \leq n-1$, since designs with $i=n$ or $i=n+1$ are classified as group divisible designs or as BIBDs. Forming the association scheme involves arranging the treatments in an $n \times n$ array, on which the Latin squares are superimposed. The first associates of a given treatment, $\tau$ say, are the $n-1$ treatments in the same array row, the $n-1$ treatments in the same array column, and, for each Latin square, the $n-1$ treatments corresponding to the same symbol as that corresponding to $\tau$. Thus, $n_{1}=i(n-1)$ and $n_{2}=(n-1)(n-i+1)$. Clatworthy (1973) listed $146 \operatorname{PBIBD}(2) \mathrm{s}$ with $L_{i}(n)$ association schemes. Cheng, Constantine \& Hedayat (1984) focussed on $L_{2}(n)$ schemes and gave a construction using bipartite subgraphs. 
From Clatworthy (1973), the association structure for a $\operatorname{PBIBD}(2)$ in the $L_{i}(n)$ category is completed by:

$$
\begin{array}{ll}
P_{1} & =\left[\begin{array}{cc}
i^{2}-3 i+n & (i-1)(n-i+1) \\
(i-1)(n-i+1) & (n-i)(n-i+1)
\end{array}\right], \\
P_{2} & = \\
{\left[\begin{array}{cc}
i(i-1) & i(n-i) \\
i(n-i) & (n-i)^{2}+i-2
\end{array}\right]}
\end{array}
$$

From (5), (6) and (8), the non-zero eigenvalues of the information matrix are $\mu_{1}=\{r(k-$ $\left.1)+i\left(\lambda_{1}-\lambda_{2}\right)+\lambda_{2}\right\} / k$ and $\mu_{2}=\left\{r(k-1)+(i-n)\left(\lambda_{1}-\lambda_{2}\right)+\lambda_{2}\right\} / k$.

Theorem 2. Every $P B I B D(2)$ with an $L_{i}(n)$ association scheme has optimal block breakdown number.

Proof: For $\lambda_{1}<\lambda_{2}$, the E-value is $\mu=\mu_{1}=\left\{r(k-1)+i\left(\lambda_{1}-\lambda_{2}\right)+\lambda_{2}\right\} / k$. From (2),

$$
\begin{aligned}
\mathcal{T} & =\frac{8 \mu k^{2}(v-k)}{v\left(2 k^{2}-1+(-1)^{k}\right)} \\
& =\frac{8\left\{r(k-1)+i\left(\lambda_{1}-\lambda_{2}\right)+\lambda_{2}\right\} k\left(n^{2}-k\right)}{n^{2}\left(2 k^{2}-1+(-1)^{k}\right)} \\
& \geq \frac{4 r(k-1)(n-i)\left(n^{2}-k\right)}{k n(n-1)(n-i+1)}
\end{aligned}
$$

using $2 k^{2}-1+(-1)^{k} \leq 2 k^{2}, \lambda_{1} \geq 0$ and $\lambda_{2} \leq \frac{r(k-1)}{(n-1)(n-i+1)}$, from (4). Furthermore, since $2 \leq i \leq n-1$ and $n \geq 3$ and for $k \in\left[2, v / 2=n^{2} / 2\right]$ the maximum value of $k+n^{2} / k$ is $2+n^{2} / 2$ :

$$
\begin{aligned}
\mathcal{T} & \geq \frac{2 r(k-1)\left(n^{2}-k\right)}{k n(n-1)} \\
& =\frac{2 r}{n(n-1)}\left\{n^{2}+1-\left(k+n^{2} / k\right)\right\} \\
& \geq \frac{r\left(n^{2}-2\right)}{n(n-1)} \geq \frac{7 r}{6}>r .
\end{aligned}
$$

For $\lambda_{1}>\lambda_{2}$, the E-value is $\mu=\mu_{2}=\left\{r(k-1)+(i-n)\left(\lambda_{1}-\lambda_{2}\right)+\lambda_{2}\right\} / k$ and (2) gives:

$$
\begin{aligned}
\mathcal{T} & =\frac{8 \mu k^{2}(v-k)}{v\left(2 k^{2}-1+(-1)^{k}\right)} \\
& =\frac{8\left\{r(k-1)+(i-n)\left(\lambda_{1}-\lambda_{2}\right)+\lambda_{2}\right\} k\left(n^{2}-k\right)}{n^{2}\left(2 k^{2}-1+(-1)^{k}\right)} \\
& \geq \frac{4 r(k-1)(i-1)\left(n^{2}-k\right)}{k n i(n-1)},
\end{aligned}
$$


using $2 k^{2}-1+(-1)^{k} \leq 2 k^{2}, \lambda_{2} \geq 0$ and $\lambda_{1} \leq \frac{r(k-1)}{i(n-1)}$, from (4). With the same approach as that used for $\lambda_{1}<\lambda_{2}$ :

$$
\mathcal{T} \geq \frac{2 r(k-1)\left(n^{2}-k\right)}{k n(n-1)}>r .
$$

Thus $\mathcal{T}>r$ for both $\lambda_{1}<\lambda_{2}$ and $\lambda_{1}>\lambda_{2}$. From (1) $t_{*}=r$, which establishes that all $\operatorname{PBIBD}(2) \mathrm{s}$ with $L_{i}(n)$ association scheme have optimal block breakdown number.

\subsection{Regular group divisible designs}

The group divisible designs form an important class of $\operatorname{PBIBD}(2) \mathrm{s}$ and have received considerable attention in the literature. The $v=m n$ treatments are divided into $m$ disjoint groups of size $n$, with $n, m \geq 2$. For clarity, the usual parameter summary is adjusted to $\left((m, n), b, r, k ;\left(\lambda_{1}, \lambda_{2}\right)\right)$ for this category. Treatments in the same group are first associates and those from different groups are second associates. The designs have $n_{1}=n-1$ and $n_{2}=n(m-1)$ and the association structure is completed by:

$$
P_{1}=\left[\begin{array}{cc}
n-2 & 0 \\
0 & n(m-1)
\end{array}\right], P_{2}=\left[\begin{array}{cc}
0 & n-1 \\
n-1 & n(m-2)
\end{array}\right] \text {. }
$$

The condition $\lambda_{2}>0$ is required for connectivity. Bose \& Connor (1952) categorised the group divisible designs into three exhaustive and mutually exclusive types: singular if $r-\lambda_{1}=0$; semi-regular if $r-\lambda_{1}>0$ and $r k-v \lambda_{2}=0$; regular if $r-\lambda_{1}>0$ and $r k-v \lambda_{2}>0$.

By an argument based on the number of pairs of first associates in treatment subsets, Godolphin \& Godolphin (2015) established that $t_{*}=r$ for all singular designs, for all semiregular designs and for regular designs with $\lambda_{1}<\lambda_{2}$. The regular designs with $\lambda_{1}>\lambda_{2}$ do not all have optimal block breakdown number. Clatworthy (1973) listed twelve regular designs with $\lambda_{1}>\lambda_{2}$ which have $t_{*}<r$. Godolphin \& Godolphin (2015) gave a lower bound for $t_{*}$ for regular designs with $\lambda_{1}>\lambda_{2}$. This is useful for assessing robustness on a design by design basis, but the design specific nature of the bound has not enabled its use in the development of general robustness conditions. Although the bound given by (1) is not specific to $\operatorname{PBIB}(2) s$, it lends itself to the development of a robustness condition for the regular designs with $\lambda_{1}>\lambda_{2}$, as is now demonstrated.

Theorem 3. Let $D$ be a regular design with $m n \geq 2 k$ and $\lambda_{1}-\lambda_{2}=\alpha$, where $\alpha \geq 1$. A sufficient condition for $D$ to have $t_{*}=r$ is $\alpha<\{(r+1)(m n-3)+2\} /\{(r-1)(n-1)\}$. 
Proof: From (6) and (9), regular designs with $\lambda_{1}>\lambda_{2}$ have $\mu=\mu_{2}=\{r(k-1)-(n-$ 1) $\left.\lambda_{1}+n \lambda_{2}\right\} / k$ and hence, from (2),

$$
\begin{aligned}
\mathcal{T} & =\frac{8\left\{r(k-1)-(n-1) \lambda_{1}+n \lambda_{2}\right\} k(m n-k)}{m n\left(2 k^{2}-1+(-1)^{k}\right)} . \\
& =\frac{8\left\{r(k-1)-\alpha(n-1)+\lambda_{2}\right\} k(m n-k)}{m n\left(2 k^{2}-1+(-1)^{k}\right)} \\
& \geq \frac{4\left\{r(k-1)-\alpha(n-1)+\lambda_{2}\right\}(m n-k)}{m n k} \\
& =\frac{4\{r(k-1)-\alpha(n-1)\}(m n-k)}{(m n-1) k}
\end{aligned}
$$

using $2 k^{2}-1+(-1)^{k} \leq 2 k^{2}, \lambda_{1}=\lambda_{2}+\alpha$ and $\lambda_{2}=\frac{r(k-1)-\alpha(n-1)}{m n-1}$, from (4). Furthermore (4) gives $r(k-1)=(m n-1) \lambda_{2}+\alpha(n-1)$ and, since $\lambda_{2} \geq 1$,

$$
r(k-1)-\alpha(n-1)=\frac{(m n-1) \lambda_{2} r(k-1)}{(m n-1) \lambda_{2}+\alpha(n-1)} \geq \frac{(m n-1) r(k-1)}{m n-1+\alpha(n-1)} .
$$

Substitution of (11) in (10) gives

$$
\mathcal{T} \geq \frac{4 r(k-1)(m n-k)}{\{m n-1+\alpha(n-1)\} k} .
$$

By elementary calculus, for $k \in[2, m n / 2]$, the minimum value of $(k-1)(m n-k) / k$ is $(m n-2) / 2$, achieved when $k=2$ and, if $m n$ is even, when $k=m n / 2$. Thus

$$
\mathcal{T} \geq \frac{2 r(m n-2)}{m n-1+\alpha(n-1)} .
$$

From (1) a sufficient condition for $D$ to have $t_{*}=r$ is given by $\mathcal{T}>r-1$. From (12) this condition is satisfied if

$$
\frac{2 r(m n-2)}{m n-1+\alpha(n-1)}>r-1,
$$

or equivalently

$$
\alpha<\frac{(r+1)(m n-3)+2}{(r-1)(n-1)},
$$

as required.

The condition specified in Theorem 3 is intuitively sensible since it suggests that for regular designs with $\lambda_{1}>\lambda_{2}$, those designs with smaller $\lambda_{1}-\lambda_{2}$ tend to be more robust against experiencing breakdown in the event of observation loss than designs with larger $\lambda_{1}-\lambda_{2}$. Thus the concurrence difference $\alpha$ can be thought of as an indicator of robustness. Significant consequences of Theorem 3 are summarised in two corollaries. 
Corollary 1. Every regular design with $\alpha=1$ has $t_{*}=r$.

Proof: Consider the right hand side of (13):

$$
\frac{(r+1)(m n-3)+2}{(r-1)(n-1)}>\frac{(r+1)(m n-3)}{(r-1)(n-1)}>\frac{m n-3}{n-1} \geq 1,
$$

since $m, n \geq 2$. Thus the right hand side of (13) exceeds one for all regular designs with $\lambda_{1}>\lambda_{2}$, and so the condition specified in Theorem 3 is satisfied for all regular designs with $\alpha=1$, confirming that every such design has $t_{*}=r$.

This result adds to the existing body of knowledge associating these designs with optimal properties. For example, John and Mitchell (1977) listed several regular designs with $\alpha=1$ as being $A, D$ and $E$ optimal in the sub-class of designs having $\left|\lambda_{1}-\lambda_{2}\right|=1$, in their respective $\mathcal{D}_{v, b, k}$ classes. Regular designs with $m=2$ and $\alpha=1$ are contained in a sub-class of designs shown by Cheng (1978) to be optimal within their $\mathcal{D}_{v, b, k}$ classes with respect to a large collection of criteria, including $A, D$ and $E$ optimality.

The second corollary can be used to confirm the optimal breakdown number for many series of regular designs with $\lambda_{1}>\lambda_{2}$.

Corollary 2. Every regular design with $m \geq 3$ and $1 \leq \alpha \leq m$ has $t_{*}=r$.

Proof: From the right hand side of (13):

$$
\frac{(r+1)(m n-3)+2}{(r-1)(n-1)}>\frac{(r+1)(m n-3)}{(r-1)(n-1)}>\frac{m n-3}{n-1} \geq m,
$$

for $m \geq 3$. Thus the condition specified in Theorem 3 is satisfied for any regular design with $m \geq 3$ and $1 \leq \alpha \leq m$ and the result follows.

Note that Corollary 2 does not apply to designs with $\alpha=2$ and $m=2$. In examinaton of the regular designs in Clatworthy (1973), Godolphin \& Godolphin (2015) identified one design with $\alpha=2$ and $m=2$ which does not have $t_{*}=r$, namely $R 2$. This indicates that Corollary 2 cannot be extended to encompass designs with $m=2$.

Corollary 2 can be illustrated by considering robustness properties of designs arising from several constructions due to Henson, Sarvate \& Hurd (2007). For simplicity some block and replication parameters are given as, say $b_{R}, r_{R}$, in the following examples. These can be obtained in terms of the other parameters of the design from (3) and (4).

Example 1 By Theorem 6 of Henson, Sarvate \& Hurd (2007), if a BIBD with parameter set $(\nu, b, r, k ; \lambda)=\left(n / 2, b_{B}, r_{B}, 4 ; m-1\right)$ exists for $n \geq 8, n \neq 12$ and $3 \leq m<n / 2$, then a regular design can be constructed with parameter set $\left((m, n), b_{R}, r_{R}, 4 ;(m-1,1)\right)$. The construction involves a pair of mutually orthogonal Latin squares of size $n / 2$. Every design has $\alpha=m-2$ and has optimal block breakdown number by Corollary 2 . 
Example 2 Adapting Theorem 9 of Henson, Sarvate \& Hurd (2007), a regular group divisible design exists with parameter set $\left((3,3), b_{R}, r_{R}, 4 ;\left(6 x_{1}+3 x_{2}+3,6 x_{1}+3 x_{2}+\right.\right.$ 1)) for all integers $x_{1}, x_{2}$, with $x_{1} \geq 1, x_{2} \geq 0$. Such designs can be constructed from $x_{1}$ copies of the group divisible design $R 104$ (see Clatworthy 1973) with parameter set $((3,3), 9,4,4 ;(3,1))$, together with $x_{1}+1$ copies of a group divisible design with parameter $\operatorname{set}((3,3), 27,12,4 ;(3,5))$ (see Figure 3 of Henson, Sarvate \& Hurd 2007) and $x_{2}$ copies of a BIBD with parameter set $(9,18,8,4 ; 3)$. Every design constructed in this manner is regular with $\alpha=2$ and $m=3$, has $t_{*}=r$ by Corollary 2 .

Example 3 By Theorem 11 of Henson, Sarvate \& Hurd (2007), a group divisible design exists with parameters $\left((5,3), b_{G}, r_{G}, 4 ;\left(x_{1}+3 x_{2}+6 x_{3}, 2 x_{1}+6 x_{3}\right)\right)$ for all $x_{1}, x_{2}, x_{3}$ non-negative integers, with at least one of $x_{1}, x_{2}, x_{3}$ positive. Consider a design with $1 \leq 3 x_{2}-x_{1} \leq 5$. The design is regular, since $r_{G}-\lambda_{1}>0$ and $4 r_{G}-15 \lambda_{2}>0$, and has $1 \leq \alpha=3 x_{2}-x_{1} \leq m$ and therefore has $t_{*}=r$ by Corollary 2 .

Bailey, R.A., Schiffl, K.\& Hilgers, R. (2013). A note on robustness of D-optimal block designs for two-colour microarray experiments. Journal of Statistical Planning and Inference 143, 1195-1202.

Baksalary, J.K. \& Tabis, Z. (1987). Conditions for the robustness of block designs against the unavailability of data. Journal of Statistical Planning and Inference 16, 49-54.

Best, J., Rayner, J.C.W. \& Allingham, D. (2011). A statistical test for ranking data from partially balanced incomplete block designs. Journal of Sensory Studies 26, 1-84.

Bose, R.C. \& Connor, W.S. (1952). Combinatorial properties of group divisible incomplete block designs. Annals of Mathematical Statistics 23, 367-383.

Bose, M., Dey, A. \& Mukerjee, R. (2013). Key predistribution schemes for distributed sensor networks via block designs. Designs, Codes and Cryptography 67, 111-136.

Clatworthy, W.H. (1973). Tables of two-associate-class partially balanced designs. National Bureau of Standards: Applied Mathematics Series 63.

Cheng, C-S. (1978). Optimality of certain asymmetrical experimental designs. Annals of Statistics 6, $1239-61$.

Cheng, C-S. (1980). On the E-optimality of some block designs. Journal of the Royal Statistical Society, Series B 42, 199-204.

Cheng, C-S. \& Bailey, R.A. (1991). Optimality of some two-associate partially balanced incomplete block designs. Annals of Statistics 19, 1667-71.

Cheng, C-S., Constantine, G.M. \& Hedayat, A.S. (1984). A unified method for constructing PBIB designs based on triangular and $L_{2}$ schemes. Journal of the Royal Statistical Society, Series B 46, 31-37.

Connor, W.S. \& Clatworthy, W.H. (1954). Some theorems for partially balanced designs. Annals of Mathematical Statistics, 25, 100-12.

Constantine, G.M. (1982). On the e-optimality of PBIB designs with a small number of blocks. Annals of Statistics 10, 1027-31.

Conniffe, D. \& Stone, J. (1975). Some incomplete block designs of maximum efficiency. Biometrika 62, 685-6. 
Ghosh, S. (1982). Robustness of BIBD against the unavailability of data. Journal of Statistical Planning and Inference 6, 29-32.

Ghosh, S., Rao, S.B. \& Singhi, N.M. (1983). On a robustness property of PBIBD. Journal of Statistical Planning and Inference 8, 355-363.

Godolphin, J.D. (2016). A link between the E-value and the robustness of block designs. Journal of the American Statistical Association 111, 1736 -1745.

Godolphin, J.D. \& Godolphin, E.J. (2015). The use of treatment concurrences to assess robustness of binary block designs against the loss of whole blocks. Australian and New Zealand Journal of Statistics 57, 225-239.

Godolphin, J.D. \& Warren, H.T. (2011). Improved conditions for the robustness of binary block designs against the loss of whole blocks. Journal of Statistical Planning and Inference 141, 3498-505.

Greig, M., Kreher, D.L. \& Ling, A. C. H. (2002). On PBIB designs based on triangular schemes. Annals of Combinatorics 6, 147-155.

Henson, D., Sarvate, D.G. \& Hurd, S.P. (2007). Group divisible designs with three groups and block size four. Discrete Mathematics 307, 1693-1706.

Hurd, S.P. \& Sarvate, D.G. (2008). Group divisible designs with block size four and two groups. Discrete Mathematics 308, 2663-2673.

Kuhn, J-T. \& Kiefer, T. (2013). Optimal Test Assembly in Practice. The Design of the Austrian Educational Standards Assessment in Mathematics. Zeitschrift für Psychologie 221, 190-200.

Mahbub Latif, A. H. M., Bretz, F. \& Brunner, E. (2009). Robustness considerations in selecting efficient two-color microarray designs. Bioinformatics 25, 2355-2361.

Sathe, Y.S. \& Satam, M.R. (1992). Some more robust block designs against the unavailability of data. Journal of Statistical Planning and Inference 30, 93-98.

Sinha, K. (1987). A method of construction of regular group divisible designs. Biometrika 74, 443-4.

Tsai, S.-F. and Liao, C.-T. (2013). Minimum breakdown designs in blocks of size two. Journal of Statistical Planning and Inference 143, 202-208. 